\title{
On the $\omega$-multiple Meixner polynomials of the first kind
}

\author{
Sonuç Zorlu Oğurlu** and IIlkay Elidemir ${ }^{1}$
}

"Correspondence:

sonuc.zorlu@emu.edu.tr

'Department of Mathematics, Eastern Mediterranean University,

Famagusta, T.R. North Cyprus, Turkey

\begin{abstract}
In this study, we introduce a new family of discrete multiple orthogonal polynomials, namely $\omega$-multiple Meixner polynomials of the first kind, where $\omega$ is a positive real number. Some structural properties of this family, such as the raising operator, Rodrigue's type formula and an explicit representation are derived. The generating function for $\omega$-multiple Meixner polynomials of the first kind is obtained and by use of this generating function we find several consequences for these polynomials. One of them is a lowering operator which will be helpful for obtaining a difference equation. We give the proof of the lowering operator by use of new technique which is a more elementary proof than the proof of Lee in (J. Approx. Theory 150:132-152, 2008). By combining the lowering operator with the raising operator we obtain the difference equation which has the $\omega$-multiple Meixner polynomials of the first kind as a solution. As a corollary we give a third order difference equation for the $\omega$-multiple Meixner polynomials of the first kind. Also it is shown that, for the special case $\omega=1$, the obtained results coincide with the existing results for multiple Meixner polynomials of the first kind. In the last section as an illustrative example we consider the special case when $\omega=1 / 2$ and, for the 1/2-multiple Meixner polynomials of the first kind, we state the corresponding result for the main theorems.
\end{abstract}

MSC: 42C05; 33E52; 33B15

Keywords: Orthogonal polynomials; Multiple orthogonal polynomials; Generating function; Difference equation

\section{Introduction}

Discrete multiple orthogonal polynomials are useful extension of discrete orthogonal polynomials, see [1-13]. The theory of discrete orthogonal polynomials on a linear lattice were extended to such polynomials by Arvesu, Coussement and Van Assche in [2]. Multiple Meixner polynomials are discrete multiple orthogonal polynomials. There are two kinds of multiple Meixner polynomials. In this paper, we concentrate on the multiple Meixner polynomials of the first kind.

Multiple Meixner polynomials of the first kind $M_{\vec{n}}^{(\beta, \vec{a})}(x)$, of degree $|\vec{n}|$, are orthogonal polynomials for the negative binomial distributions when $r>1$. That is,

$$
\sum_{x=0}^{\infty} M_{\vec{n}}^{(\beta, \vec{a})}(x)(-x)_{j} w_{i}^{\beta}(x)=0, \quad j=0,1, \ldots, n_{i}-1, i=1, \ldots, r,
$$

(c) The Author(s) 2020. This article is licensed under a Creative Commons Attribution 4.0 International License, which permits use, sharing, adaptation, distribution and reproduction in any medium or format, as long as you give appropriate credit to the original author(s) and the source, provide a link to the Creative Commons licence, and indicate if changes were made. The images or other third party material in this article are included in the article's Creative Commons licence, unless indicated otherwise in a credit line to the material. If material is not included in the article's Creative Commons licence and your intended use is not permitted by statutory regulation or exceeds the permitted use, you will need to obtain permission directly from the copyright holder. To view a copy of this licence, visit http://creativecommons.org/licenses/by/4.0/. 
where $\beta>0$ is the fixed parameter and different values for the parameter $\vec{a}=\left(a_{1}, \ldots, a_{r}\right)$, $\left(a_{i} \neq a_{j}\right.$ whenever $\left.i \neq j\right)$, where $0<a<1$ with multi-index $\vec{n}=\left(n_{1}, \ldots, n_{r}\right)$ and $(x)_{k}=x(x+$ 1) $(x+2) \cdots(x+k-1)$ is the Pochhammer symbol with $(x)_{0}=1$.

The functions

$$
w_{i}^{\beta}(x)= \begin{cases}\frac{\Gamma(\beta+x) a_{i}^{k}}{\Gamma(\beta) \Gamma(x+1)} & \text { if } x \in \mathbb{R} /(\{-1,-2, \ldots\} \cup\{-\beta,-\beta-1, \ldots\}), \\ 0 & \text { if } x \in\{-1,-2, \ldots\},\end{cases}
$$

are weight functions of the multiple Meixner polynomials of the first kind where $\Gamma(x)$ is the gamma function.

The orthogonality conditions give us a linear system of $|\vec{n}|=n_{1}+n_{2}+\cdots+n_{r}$ homogeneous equations for the $|\vec{n}|+1$ unknown coefficients of polynomials which always has a nontrivial solution. If the given multi-index $\vec{n}$ is normal, then the corresponding polynomials will be unique polynomials. For the uniqueness of the polynomials one can use a system called an AT system which was introduced by Nikishin and Sorokin [11].

Definition 1.1 (cf. [11]) A set of continuous real functions $w_{1}, w_{2}, \ldots, w_{r}$ defined on $[a, b]$ is called an AT system for the index $n \in \mathbb{Z}_{+}^{r}, n \neq 0$, if

$$
w_{1}(x), x w_{1}(x), \ldots, x^{n_{1}-1} w_{1}(x), \ldots, w_{r}(x), x w_{r}(x), \ldots, x^{n_{r}-1} w_{r}(x)
$$

is a Chebyshev system of order $|n|-1$ on $[a, b]$.

In an AT system, all the multi-index $\vec{n}$ are normal. By using the following example it will be easy to show that the weight functions for the multiple Meixner polynomials of the first kind form an AT system.

Example 1.2 (cf. [2]) The functions $w(x) a_{1}^{x}, x w(x) a_{1}^{x}, \ldots, x^{n_{1}-1} w(x) a_{1}^{x}, \ldots, w(x) a_{r}^{x}, x w(x) a_{r}^{x}$, $\ldots, x^{n_{r}-1} w(x) a_{r}^{x}$, with all the $a_{i}>0, i=1, \ldots, r$, different and $w(x)$ a continuous function which has no zeros on $\mathbb{R}^{+}$, form a Cheybshev system on $\mathbb{R}^{+}$for every index $\vec{n} \in \mathbb{N}^{r}$.

In [2], Arvesu, Coussement and Van Assche investigated the raising operator and the Rodrigues formula for multiple Meixner polynomials of the first kind. Also, via the Rodrigues formula an explicit formula for these polynomials is obtained by these authors. They investigated these properties for multiple orthogonal polynomials of discrete variables by extending the classical orthogonal polynomials of discrete variables.

Van Assche in [12] obtained a lowering operator for multiple Meixner polynomials of the first kind for the case $r=2$ and then by combining lowering and raising operators he gave the third order difference equation for these polynomials. Later, Lee in [7] obtained a lowering operator for the case $r$ and then by combining lowering and raising operators Lee gave the $(r+1)$ th order difference equation for these polynomials.

Ndayiragije and Van Assche in [4] gave generating functions and explicit expressions for the coefficients in the nearest neighbor recurrence relation for multiple Meixner polynomials of the first kind.

In this paper we introduce a new class for discrete multiple polynomials called $\omega$ multiple Meixner polynomials of the first kind. The aim is to obtain some structural properties for these newly introduced polynomials. Firstly we define the orthogonality for the 
$\omega$-multiple Meixner polynomials of the first kind on the linear lattice by using two important operators, namely the $\omega$-forward and $\omega$-backward difference operators, where

$$
\triangle_{\omega} f(x)=f(x+\omega)-f(x)
$$

is the $\omega$-forward operator and

$$
\nabla_{\omega} f(x)=f(x)-f(x-\omega)
$$

is the $\omega$-backward operator.

In Sect. 2 we obtain some properties of the $\omega$-multiple Meixner polynomials of the first kind, such as the raising operator, Rodrigues' formula and an explicit form. The generating function for the $\omega$-multiple Meixner polynomials of the first kind is given in Sect. 3 and we obtain some results from the generating function such as connection and addition formulas. Section 4 includes the lowering operator and difference equation for the $\omega$-multiple Meixner polynomials of the first kind. We also show that when $\omega=1$, the results obtained in Sects. 2, 3 and 4, coincide with the existing results for multiple Meixner polynomials of the first kind. One of the main results of this paper is in Sect. 5 where we give some results for the 1/2-multiple Meixner polynomials of the first kind.

\section{Orthogonality for $\omega$-multiple Meixner polynomials of the first kind}

In this section, we first give the definition of the orthogonality of $\omega$-multiple Meixner polynomials of the first kind.

Definition 2.1 The monic discrete $\omega$-multiple Meixner polynomial of the first kind, corresponding to the multi-index $\vec{n}=\left(n_{1}, \ldots, n_{r}\right)$, the fixed parameter $\beta>0$ and the parameter $\vec{a}=\left(a_{1}, \ldots, a_{r}\right),\left(a_{i} \neq a_{j}\right.$ whenever $\left.i \neq j\right)$, is the unique polynomial of degree $|\vec{n}|$ which satisfies the orthogonality conditions

$$
\sum_{x=0}^{\infty} M_{\vec{n}}^{(\omega ; \beta ; \vec{a})}(\omega x)(-\omega x)_{j, \omega} w_{i}^{(\omega ; \beta)}(\omega x)=0, \quad j=0,1, \ldots, n_{i}-1, i=1,2, \ldots, r
$$

where $(a)_{n, \omega}=a(a+\omega)(a+2 \omega) \cdots(a+(n-1) \omega)$ is the $\omega$-Pochhammer symbol for $a \in \mathbb{C}$ and $n \in \mathbb{N}$ and $\omega>0$.

The weight functions for $\omega$-multiple Meixner polynomials of the first kind are defined as

$$
w_{i}^{(\omega ; \beta)}(x)= \begin{cases}\frac{\Gamma_{\omega}(\beta+x) a_{i}^{x}}{\Gamma_{\omega}(\beta) \Gamma_{\omega}(x+\omega)} & \text { if } x \in \mathbb{R} /(\{-1,-2, \ldots\} \cup\{-\beta,-\beta-1, \ldots\}), \\ 0 & \text { if } x \in\{-1,-2, \ldots\}\end{cases}
$$

where $0<a_{i}<1$, for $i=, 1,2, \ldots, r$, with all the $a_{i}$ different and $\Gamma_{\omega}$ is the $\omega$-gamma function given by

$$
\Gamma_{\omega}(x)=\int_{0}^{\infty} t^{x-1} e^{-\frac{t^{\omega}}{\omega}} d t=\omega^{\frac{x}{\omega}-1} \Gamma\left(\frac{x}{\omega}\right)
$$


By Example 1.2 it is easy to conclude that the weight functions form an AT system which implies the uniqueness of such polynomials. When $\omega=1$, the given orthogonality conditions coincide with the orthogonality conditions in (1).

In the rest of this paper the properties of multiple Meixner polynomials of the first kind are extended to $\omega$-multiple Meixner polynomials of the first kind.

Theorem 2.2 Let $\omega$ be a positive real number. The raising operator for $\omega$-multiple Meixner polynomials of the first kind is given as

$$
\nabla_{\omega}\left[M_{\vec{n}}^{(\omega ; \beta ; \vec{a})}(x) w_{i}^{(\omega ; \beta)}(x)\right]=\frac{a_{i}^{\omega}-1}{a_{i}^{\omega}(\beta-\omega)} M_{\vec{n}+\vec{e}_{i}}^{(\omega ; \beta-\omega ; \vec{a})}(x) w_{i}^{(\omega ; \beta-\omega)}(x)
$$

Proof By using the product rule for the $\omega$-backward operator

$$
\nabla_{\omega}[f(x) g(x)]=f(x) \nabla_{\omega} g(x)+g(x-\omega) \nabla_{\omega} f(x) \text {, we obtain }
$$

$$
\nabla_{\omega}\left[M_{\vec{n}}^{(\omega ; \beta ; \vec{a})}(x) w_{i}^{(\omega ; \beta)}(x)\right]=\frac{a_{i}^{\omega}-1}{a_{i}^{\omega}(\beta-\omega)}(x) w_{i}^{(\omega ; \beta-\omega)}(x) P_{\vec{n}+\vec{e}_{i}}^{(\omega)} .
$$

Using the $\omega$-summation by parts formula,

$$
\sum_{k=0}^{\infty} \triangle_{\omega}[f(\omega k)] g(\omega k)=-\sum_{k=0}^{\infty} \nabla_{\omega}[g(\omega k)] f(\omega k) \quad \text { where } g(-\omega)=0
$$

and the orthogonality conditions,

$$
\sum_{k=0}^{\infty} P_{\vec{n}+\vec{e}_{i}}^{(\omega)}(\omega x)(-\omega x)_{j, \omega} w_{i}^{(\omega ; \beta-\omega)}(\omega x)=0
$$

we find the result with $P_{\vec{n}+\overrightarrow{e_{i}}}^{(\omega)}=M_{\vec{n}+\overrightarrow{e_{i}}}^{(\omega ; \beta-\omega ; \vec{a})}$, which was guaranteed from the uniqueness of the orthogonal polynomials.

Theorem 2.3 Let $\omega$ be a positive real number. The Rodrigues formula for $\omega$-multiple Meixner polynomials of the first kind are introduced by

$$
\begin{aligned}
M_{\vec{n}}^{(\omega ; \beta ; \vec{a})}(x)= & (\beta)_{|\vec{n}|, \omega}\left[\prod_{k=1}^{r}\left(\frac{a_{k}^{\omega}}{a_{k}^{\omega}-1}\right)^{n_{k}} \frac{\Gamma_{\omega}(\beta) \Gamma_{\omega}(x+\omega)}{\Gamma_{\omega}(\beta+x)}\right] \\
& \times \prod_{i=1}^{r} \frac{1}{a_{i}^{x}} \nabla_{\omega}^{n_{i}}\left[\frac{\Gamma_{\omega}(\beta+x+|\vec{n}| \omega) a_{i}^{x}}{\Gamma_{\omega}(x+\omega) \Gamma_{\omega}(\beta+|\vec{n}| \omega)}\right] .
\end{aligned}
$$

Proof Replacing $\vec{n}$ by $\vec{n}-\overrightarrow{e_{i}}$ and $\beta$ by $\beta+\omega$ in the raising operator formula, we obtain

$$
w_{i}^{(\omega ; \beta)}(x) M_{\vec{n}}^{(\omega ; \beta ; \vec{a})}(x)=\frac{\beta a_{i}^{\omega}}{a_{i}^{\omega}-1} \nabla_{\omega}\left[w_{i}^{(\omega ; \beta+\omega)}(x) M_{\vec{n}-\overrightarrow{e_{i}}}^{(\omega ; \beta+\omega ; \vec{a})}(x)\right] .
$$

Then for $r=2$ the multi-index will be $\vec{n}=\left(n_{1}, n_{2}\right)$.

For $i=1$ we have

$$
w_{1}^{(\omega ; \beta)}(x) M_{n_{1}, n_{2}}^{\left(\omega ; \beta ; a_{1}, a_{2}\right)}(x)=\frac{\beta a_{1}^{\omega}}{a_{1}^{\omega}-1} \nabla_{\omega}\left[w_{1}^{(\omega ; \beta+\omega)}(x) M_{n_{1}-1, n_{2}}^{\left(\omega ; \beta+\omega ; a_{1}, a_{2}\right)}(x)\right],
$$


and iterating it $n_{1}$ times we get

$$
\begin{aligned}
w_{1}^{((\omega ; \beta)}(x) M_{n_{1}, n_{2}}^{\left(\omega ; ; ; a_{1}, a_{2}\right)}(x)= & (\beta)_{n_{1}, \omega}\left(\frac{a_{1}^{\omega}}{a_{1}^{\omega}-1}\right)^{n_{1}} \\
& \times \nabla_{\omega}^{n_{1}}\left[w_{1}^{\left(\omega ; \beta+n_{1} \omega\right)}(x) M_{0, n_{2}}^{\left(\omega ; \beta+n_{1} \omega ; a_{1}, a_{2}\right)}(x)\right] .
\end{aligned}
$$

For $i=2$ we have

$$
w_{2}^{(\omega ; \beta)}(x) M_{n_{1}, n_{2}}^{\left(\omega ; \beta ; a_{1}, a_{2}\right)}(x)=\frac{\beta a_{2}^{\omega}}{a_{2}^{\omega}-1} \nabla_{\omega}\left[w_{2}^{(\omega ; \beta+\omega)}(x) M_{n_{1}, n_{2}-1}^{\left(\omega ; \beta+\omega ; a_{1}, a_{2}\right)}(x)\right],
$$

and iterating it $n_{2}$ times we get

$$
\begin{aligned}
w_{2}^{(\omega ; \beta)}(x) M_{n_{1}, n_{2}}^{\left(\omega ; \beta ; a_{1}, a_{2}\right)}(x)= & (\beta)_{n_{2}, \omega}\left(\frac{a_{2}^{\omega}}{a_{2}^{\omega}-1}\right)^{n_{2}} \\
& \times \nabla_{\omega}^{n_{2}}\left[w_{1}^{\left(\omega ; \beta+n_{2} \omega\right)}(x) M_{n_{1}, 0}^{\left(\omega ; \beta+n_{2} \omega ; a_{1}, a_{2}\right)}(x)\right] .
\end{aligned}
$$

By combining these two equations, we obtain the expression for $M_{n_{1}, n_{2}}^{\left(\omega ; \beta a_{1}, a_{2}\right)}(x)$ and if we continue the iteration for $r$, we derive the Rodrigues formula for $\omega$-multiple Meixner polynomials of the first kind.

The explicit form can easily be obtained from the Rodrigues formula using the Leibniz rule for the $\omega$-backward operator,

$$
\nabla_{\omega}^{n} f(x)=\sum_{i=1}^{n}(-1)^{i}\left(\begin{array}{c}
n \\
i
\end{array}\right) f(x-i \omega)
$$

Theorem 2.4 Let $\omega$ be a positive real number. The explicit form for $\omega$-multiple Meixner polynomials of the first kind is given by

$$
\begin{aligned}
M_{\vec{n}}^{(\omega ; \beta ; \vec{a})}(x)= & \sum_{k_{1}=0}^{n_{1}} \sum_{k_{2}=0}^{n_{2}} \cdots \sum_{k_{r}=0}^{n_{r}}\left(\begin{array}{l}
n_{1} \\
k_{1}
\end{array}\right)\left(\begin{array}{l}
n_{2} \\
k_{2}
\end{array}\right) \ldots\left(\begin{array}{l}
n_{r} \\
k_{r}
\end{array}\right)(-x)_{|\vec{k}|, \omega} \\
& \times \prod_{j=1}^{r}\left[\frac{\left(a_{j}^{\omega}\right)^{n_{j}-k_{j}}}{\left(a_{j}^{\omega}-1\right)^{n_{j}}}(\beta+x)_{|\vec{n}|-|\vec{k}|, \omega}\right] .
\end{aligned}
$$

Corollary 2.5 The special cases of Theorem 2.2, Theorem 2.3 and Theorem 2.4 when $\omega=1$ are easily reduced to results obtained in [2] and [4]. For instance, when $\omega=1$ in Theorem 2.2, our result coincides with the raising operator for multiple Meixner polynomials of the first kind,

$$
\nabla\left[M_{\vec{n}}^{(\beta, \vec{a})}(x) w_{i}^{\beta}(x)\right]=\frac{a_{i}-1}{a_{i}(\beta-1)} M_{\vec{n}+\vec{e}_{i}}^{(\beta-1, \vec{a})}(x) w_{i}^{\beta-1}(x),
$$

which is exactly the same formula as in [2, equation (4.6), $p$. 33].

When $\omega=1$ in Theorem 2.3, we find the Rodrigues formula for multiple Meixner polynomials of the first kind,

$$
M_{\vec{n}}^{(\beta, \vec{a})}(x)=(\beta)_{|\vec{n}|}\left[\prod_{k=1}^{r}\left(\frac{a_{k}}{a_{k}-1}\right)^{n_{k}} \frac{\Gamma(\beta) \Gamma(x+1)}{\Gamma(\beta+x)}\right]
$$




$$
\times \prod_{i=1}^{r} \frac{1}{a_{i}^{x}} \nabla^{n_{i}}\left[\frac{\Gamma(\beta+x+|\vec{n}|) a_{i}^{x}}{\Gamma(x+1) \Gamma(\beta+|\vec{n}|)}\right]
$$

which coincides with the formula in [2, equation (4.7), p. 33].

When $\omega=1$ in Theorem 2.4, we have an explicit form for multiple Meixner polynomials of the first kind,

$$
\begin{aligned}
M_{\vec{n}}^{(\beta, \vec{a})}(x)= & \sum_{k_{1}=0}^{n_{1}} \sum_{k_{2}=0}^{n_{2}} \cdots \sum_{k_{r}=0}^{n_{r}}\left(\begin{array}{l}
n_{1} \\
k_{1}
\end{array}\right)\left(\begin{array}{l}
n_{2} \\
k_{2}
\end{array}\right) \cdots\left(\begin{array}{l}
n_{r} \\
k_{r}
\end{array}\right)(-x)_{|\vec{k}|} \\
& \times \prod_{j=1}^{r}\left[\frac{\left(a_{k}\right)^{n_{j}-k_{j}}}{\left(a_{k}-1\right)^{n_{j}}}(\beta+x)_{|\vec{n}|-|\vec{k}|}\right],
\end{aligned}
$$

which coincides with the formula in [4, equation (3), p. 3]

\section{Generating function for $\omega$-multiple Meixner polynomials of the first kind}

$\omega$-Multiple Meixner polynomials of the first kind have a multivariate generating function with $r$ variables. The following lemma will be useful for the proof of the theorem for the generating function.

The relation between Pochhammer symbol and $\omega$-Pochhammer symbol is given as follows:

$$
(a)_{n, \omega}=\omega^{n}\left(\frac{a}{\omega}\right)_{n}
$$

Lemma 3.1 (cf. [4, Lemma 1, p. 4]) The generating function for the multinomial coefficients is given as follows:

$$
\sum_{m_{1}=0}^{\infty} \cdots \sum_{m_{r}=0}^{\infty} \frac{(-x)_{|\vec{m}|}}{m_{1} ! \cdots m_{r} !} t_{1}^{m_{1}} \cdots t_{r}^{m_{r}}=\left(1-t_{1} \cdots-t_{r}\right)^{x} .
$$

This series converges absolutely and uniformly for $\left|t_{1}\right|+\cdots+\left|t_{r}\right|<1$ when $x \notin \mathbb{N}$ and contains a finite number of terms if $x \in \mathbb{N}$.

Theorem 3.2 Let $\omega$ be a positive real number. The generating function for the $\omega$-multiple Meixner polynomials of the first kind is

$$
\begin{aligned}
\sum_{n_{1}=0}^{\infty} \sum_{n_{2}=0}^{\infty} \cdots \sum_{n_{r}=0}^{\infty} M_{\vec{n}}^{(\omega ; \beta ; \vec{a})}(x) \frac{t_{1}^{n_{1}} \cdots t_{r}^{n_{r}}}{n_{1} ! \cdots n_{r} !}= & \left(1-\frac{\omega t_{1}}{a_{1}^{\omega}-1}-\cdots-\frac{\omega t_{r}}{a_{r}^{\omega}-1}\right)^{\frac{x}{\omega}} \\
& \times\left(1-\frac{\omega t_{1} a_{1}^{\omega}}{a_{1}^{\omega}-1}-\cdots-\frac{\omega t_{r} a_{r}^{\omega}}{a_{r}^{\omega}-1}\right)^{\frac{-(\beta+x)}{\omega}} .
\end{aligned}
$$

Proof Replacing $M_{\vec{n}}^{(\omega ; \beta ; \vec{a})}(x)$ with the explicit form in left hand side of (7) we obtain

$$
\sum_{n_{1}=0}^{\infty} \cdots \sum_{n_{r}=0}^{\infty} \sum_{k_{1}=0}^{n_{1}} \cdots \sum_{k_{r}=0}^{n_{r}}\left(\begin{array}{l}
n_{1} \\
k_{1}
\end{array}\right)\left(\begin{array}{l}
n_{2} \\
k_{2}
\end{array}\right) \cdots\left(\begin{array}{c}
n_{r} \\
k_{r}
\end{array}\right)(-x)_{|\vec{k}|, \omega}
$$




$$
\times \prod_{j=1}^{r}\left[\frac{\left(a_{k}^{\omega}\right)^{n_{j}-k_{j}}}{\left(a_{k}^{\omega}-1\right)^{n_{j}}}(\beta+x)_{|\vec{n}|-|\vec{k}|, \omega}\right] \frac{t_{1}^{n_{1}} \cdots t_{r}^{n_{r}}}{n_{1} ! \cdots n_{r} !} .
$$

Changing the order of the summation gives

$$
\begin{aligned}
& \sum_{k_{1}=0}^{\infty} \cdots \sum_{k_{r}=0}^{\infty} \sum_{n_{1}=k_{1}}^{\infty} \cdots \sum_{n_{r}=k_{r}}^{\infty} \frac{(-x)|\vec{k}|, \omega}{k_{1} !\left(n_{1}-k_{1}\right) ! \cdots k_{r} !\left(n_{r}-k_{r}\right) !} \frac{a_{1}^{\omega\left(n_{1}-k_{1}\right)} \cdots a_{r}^{\omega\left(n_{r}-k_{r}\right)}}{\left(a_{1}^{\omega}-1\right)^{n_{1}} \cdots\left(a_{r}^{\omega}-1\right)^{n_{r}}} \\
& \quad \times x t_{1}^{n_{1}} \cdots t_{r}^{n_{r}}(\beta+x)_{|\vec{n}|-|\vec{k}|, \omega}
\end{aligned}
$$

By setting $m_{i}=n_{i}-k_{i}$ and putting the factors in $m_{i}$ and $k_{i}$ together we obtain

$$
\begin{aligned}
& \sum_{k_{1}=0}^{\infty} \cdots \sum_{k_{r}=0}^{\infty} \frac{(-x)|\vec{k}|, \omega}{k_{1} ! \cdots k_{r} !}\left(\frac{t_{1}}{a_{1}^{\omega}-1}\right)^{k_{1}} \cdots\left(\frac{t_{r}}{a_{r}^{\omega}-1}\right)^{k_{r}} \\
& \quad \times \sum_{m_{1}=0}^{\infty} \cdots \sum_{m_{r}=0}^{\infty} \frac{(\beta+x)_{|\vec{m}|, \omega}}{m_{1} ! \cdots m_{r} !}\left(\frac{t_{1} a_{1}^{\omega}}{a_{1}^{\omega}-1}\right)^{m_{1}} \cdots\left(\frac{t_{r} a_{r}^{\omega}}{a_{r}^{\omega}-1}\right)^{m_{r}} .
\end{aligned}
$$

Using the relation between the Pochhammer symbol and the $\omega$-Pochhammer symbol (6), the above equation becomes

$$
\begin{aligned}
& \sum_{k_{1}=0}^{\infty} \cdots \sum_{k_{r}=0}^{\infty} \frac{\left(\frac{-x}{\omega}\right)|\vec{k}|}{k_{1} ! \cdots k_{r} !}\left(\frac{\omega t_{1}}{a_{1}^{\omega}-1}\right)^{k_{1}} \cdots\left(\frac{\omega t_{r}}{a_{r}^{\omega}-1}\right)^{k_{r}} \\
& \quad \times \sum_{m_{1}=0}^{\infty} \cdots \sum_{m_{r}=0}^{\infty} \frac{\left(\frac{\beta+x}{\omega}\right)_{|\vec{m}|}}{m_{1} ! \cdots m_{r} !}\left(\frac{\omega t_{1} a_{1}^{\omega}}{a_{1}^{\omega}-1}\right)^{m_{1}} \cdots\left(\frac{\omega t_{r} a_{r}^{\omega}}{a_{r}^{\omega}-1}\right)^{m_{r}} .
\end{aligned}
$$

Now using Lemma 3.1, we obtain the generating function for $\omega$-multiple Meixner polynomials of the first kind, which is the desired result.

Corollary 3.3 When $\omega=1$, the special case of Theorem 3.2 reduces to the result of [4]. For instance, when $\omega=1$ in Theorem 3.2, we have a generating function for multiple Meixner polynomials of the first kind,

$$
\begin{aligned}
\sum_{n_{1}=0}^{\infty} \sum_{n_{2}=0}^{\infty} \cdots \sum_{n_{r}=0}^{\infty} M_{\vec{n}}^{(\beta, \vec{a})}(x) \frac{t_{1}^{n_{1}} \cdots t_{r}^{n_{r}}}{n_{1} ! \cdots n_{r} !}= & \left(1-\frac{t_{1}}{a_{1}-1}-\cdots-\frac{t_{r}}{a_{r}-1}\right)^{x} \\
& \times\left(1-\frac{t_{1} a_{1}}{a_{1}-1}-\cdots-\frac{t_{r} a_{r}}{a_{r}-1}\right)^{-(\beta+x)},
\end{aligned}
$$

which coincides with the formula in [4, equation (7), p. 4].

The generating function will be used to establish the connection formula and the addition formula for $\omega$-multiple Meixner polynomials of the first kind. 
Theorem 3.4 Let $\omega$ be a positive real number. $\omega$-Multiple Meixner polynomials of the first kind $M_{\vec{n}}^{(\omega ; \beta ; \vec{a})}(x)$ and $M_{\vec{n}-\vec{k}}^{(\omega ; \beta+\gamma ; \vec{a})}(x)$ satisfy the following connection formula:

$$
\begin{aligned}
M_{\vec{n}}^{(\omega ; \beta ; \vec{a})}(x)= & \sum_{k_{1}=0}^{n_{1}} \cdots \sum_{k_{r}=0}^{n_{r}}\left(\begin{array}{l}
n_{1} \\
k_{1}
\end{array}\right) \cdots\left(\begin{array}{c}
n_{r} \\
k_{r}
\end{array}\right)(-\gamma)_{|\vec{k}|, \omega} \\
& \times\left(\frac{a_{1}^{\omega}}{a_{1}^{\omega-1}}\right)^{k_{1}} \cdots\left(\frac{a_{r}^{\omega}}{a_{r}^{\omega-1}}\right)^{k_{r}} M_{\vec{n}-\vec{k}}^{(\omega ; \beta+\gamma ; \vec{a})}(x) .
\end{aligned}
$$

Proof Replacing $\beta$ by $\beta-\gamma+\gamma$ in the generating function (7) we obtain

$$
\begin{aligned}
\sum_{n_{1}=0}^{\infty} \cdots \sum_{n_{r}=0}^{\infty} M_{\vec{n}}^{(\omega ; \beta ; \vec{a})}(x) \frac{t_{1}^{n_{1}} \cdots t_{r}^{n_{r}}}{n_{1} ! \cdots n_{r} !}= & \left(1-\frac{\omega t_{1}}{a_{1}^{\omega}-1}-\cdots-\frac{\omega t_{r}}{a_{r}^{\omega}-1}\right)^{\frac{x}{\omega}} \\
& \times\left(1-\frac{\omega t_{1} a_{1}^{\omega}}{a_{1}^{\omega}-1}-\cdots-\frac{\omega t_{r} a_{r}^{\omega}}{a_{r}^{\omega}-1}\right)^{\frac{-(\beta+\gamma+x)}{\omega}} \\
& \times\left(1-\frac{\omega t_{1} a_{1}^{\omega}}{a_{1}^{\omega}-1}-\cdots-\frac{\omega t_{r} a_{r}^{\omega}}{a_{r}^{\omega}-1}\right)^{\frac{\gamma}{\omega}}
\end{aligned}
$$

From the generating function (7) and Lemma 3.1, the above equation gets the following form:

$$
\begin{aligned}
\sum_{n_{1}=0}^{\infty} \cdots \sum_{n_{r}=0}^{\infty} M_{\vec{n}}^{(\omega ; \beta ; \vec{a})}(x) \frac{t_{1}^{n_{1}} \cdots t_{r}^{n_{r}}}{n_{1} ! \cdots n_{r} !}= & \sum_{n_{1}=0}^{\infty} \cdots \sum_{n_{r}=0}^{\infty} M_{\vec{n}}^{(\beta+\gamma, \vec{a})}(x) \frac{t_{1}^{n_{1}} \cdots t_{r}^{n_{r}}}{n_{1} ! \cdots n_{r} !} \\
& \times \sum_{k_{1}=0}^{\infty} \cdots \sum_{k_{r}=0}^{\infty} \frac{\left(\frac{-\gamma}{\omega}\right)}{k_{1} ! \cdots k_{r} !}\left(\frac{\omega a_{1}^{\omega}}{a_{1}^{\omega}-1}\right)^{k_{1}} \cdots\left(\frac{\omega a_{r}^{\omega}}{a_{r}^{\omega}-1}\right)^{k_{r}} \\
& \times t_{1}^{k_{1}} \cdots t_{r}^{k_{r}} .
\end{aligned}
$$

Changing the order of summations and using the relation between the Pochhammer symbol and the $\omega$-Pochhammer symbol (6) we get

$$
\begin{aligned}
\sum_{n_{1}=0}^{\infty} \cdots \sum_{n_{r}=0}^{\infty} M_{\vec{n}}^{(\omega ; \beta ; \vec{a})}(x) \frac{t_{1}^{n_{1}} \cdots t_{r}^{n_{r}}}{n_{1} ! \cdots n_{r} !}= & \sum_{n_{1}=0}^{\infty} \cdots \sum_{n_{r}=0}^{\infty} \sum_{k_{1}=0}^{n_{1}} \cdots \sum_{k_{r}=0}^{n_{r}}\left(\begin{array}{c}
n_{1} \\
k_{1}
\end{array}\right) \cdots\left(\begin{array}{c}
n_{r} \\
k_{r}
\end{array}\right) \\
& \times M_{\vec{n}-\vec{k}}^{(\omega ; \beta+\gamma ; \vec{a})}(x)(-\gamma)_{|\vec{k}|, \omega}\left(\frac{a_{1}^{\omega}}{a_{1}^{\omega-1}}\right)^{k_{1}} \cdots \\
& \times\left(\frac{a_{r}^{\omega}}{a_{r}^{\omega-1}}\right)^{k_{r}} \frac{t_{1}^{n_{1}} \cdots t_{r}^{n_{r}}}{n_{1} ! \cdots n_{r} !}
\end{aligned}
$$

Finally, comparing the coefficients of $\frac{t_{1}^{n_{1}} \ldots r_{r}^{n_{r}}}{n_{1} ! \cdots n_{r} !}$, appearing on both sides of the above equation, we obtain the desired result. 
Theorem 3.5 Let $\omega$ be a positive real number. The addition formula for $\omega$-multiple Meixner polynomials of the first kind is given by

$$
M_{\vec{n}}^{(\omega ; \beta+\gamma ; \vec{a})}(x+y)=\sum_{k_{1}=0}^{n_{1}} \cdots \sum_{k_{r}=0}^{n_{r}}\left(\begin{array}{c}
n_{1} \\
k_{1}
\end{array}\right) \cdots\left(\begin{array}{c}
n_{r} \\
k_{r}
\end{array}\right) M_{\vec{n}-\vec{k}}^{(\omega ; \beta ; \vec{a})}(x) M_{\vec{k}}^{(\omega ; \gamma ; \vec{a})}(y) .
$$

Proof Changing $x$ with $x+y$ and $\beta$ with $\beta+\gamma$ in the generating function (7), together with changing the order of summations we obtain

$$
\begin{aligned}
\sum_{n_{1}=0}^{\infty} \cdots \sum_{n_{r}=0}^{\infty} M_{\vec{n}}^{(\beta+\gamma, \vec{a})}(x+y) \frac{t_{1}^{n_{1}} \cdots t_{r}^{n_{r}}}{n_{1} ! \cdots n_{r} !}= & \sum_{n_{1}=0}^{\infty} \cdots \sum_{n_{r}=0}^{\infty} \sum_{k_{1}=0}^{n_{1}} \cdots \sum_{k_{r}=0}^{n_{r}} \frac{t_{1}^{k_{1}} \cdots t_{r}^{k_{r}}}{k_{1} ! \cdots k_{r} !} M_{\vec{k}}^{(\gamma, \vec{a})}(y) \\
& \times \frac{t_{1}^{n_{1}-k_{1}} \cdots t_{r}^{n_{r}-k_{r}}}{\left(n_{1}-k_{1}\right) ! \cdots\left(n_{r}-k_{r}\right) !} M_{\vec{n}-\vec{k}}^{(\beta, \vec{a})}(x) .
\end{aligned}
$$

Finally, comparing the coefficients of $\frac{t_{1}^{n_{1} \ldots t_{r} n_{r}}}{n_{1} ! \cdots n_{r} !}$, appearing on both sides of the above equation we get the result.

Note that in the case when $\omega=1$, the connection and addition formula for multiple Meixner polynomials are also new. So, here we have new relations for multiple Meixner polynomials of the first kind, which are given below.

Corollary 3.6 Multiple Meixner polynomials of the first kind satisfy the following connection and addition formulas, respectively:

$$
\begin{aligned}
& M_{\vec{n}}^{(\beta, \vec{a})}(x)=\sum_{k_{1}=0}^{n_{1}} \cdots \sum_{k_{r}=0}^{n_{r}}\left(\begin{array}{l}
n_{1} \\
k_{1}
\end{array}\right) \cdots\left(\begin{array}{l}
n_{r} \\
k_{r}
\end{array}\right) a_{1}^{k_{1}} \cdots a_{r}^{k_{r}}(-\gamma)_{|\vec{k}|} M_{\vec{n}-\vec{k}}^{(\beta+\gamma, \vec{a})}(x), \\
& M_{\vec{n}}^{(\beta+\gamma, \vec{a})}(x+y)=\sum_{k_{1}=0}^{n_{1}} \cdots \sum_{k_{r}=0}^{n_{r}}\left(\begin{array}{l}
n_{1} \\
k_{1}
\end{array}\right) \cdots\left(\begin{array}{c}
n_{r} \\
k_{r}
\end{array}\right) M_{\vec{n}-\vec{k}}^{(\beta, \vec{a})}(x) M_{\vec{k}}^{(\gamma, \vec{a})}(y) .
\end{aligned}
$$

\section{Difference equation for $\omega$-multiple Meixner polynomials of the first kind}

In this section, the aim is to introduce the difference equation for the $\omega$-multiple Meixner polynomials of the first kind by combining the lowering and raising operators.

Theorem 4.1 Let $\omega$ be a positive real number. The raising operator for $\omega$-multiple Meixner polynomials of the first kind is given as

$$
L_{a_{i}}^{(\beta)}\left[M_{\vec{n}}^{(\omega ; \beta ; \vec{a})}\right]=-M_{\vec{n}+e_{i}}^{(\omega ; \beta-\omega ; \vec{a})}
$$

where $L_{a_{i}}^{(\beta)}[\cdot]$ is defined by

$$
L_{a_{i}}^{(\beta)}[y]=\frac{x}{1-a_{i}^{\omega}} \nabla_{\omega} y-\left[\frac{a_{i}^{\omega}(\beta-\omega)}{1-a_{i}^{\omega}}-x\right] y .
$$

Proof The proof follows directly from the Rodrigues formula (4) for the $\omega$-multiple Meixner polynomials of the first kind. 
Theorem 4.2 Let $\omega$ be a positive real number. The lowering operator for $\omega$-multiple Meixner polynomials of the first kind is

$$
\Delta_{\omega} M_{\vec{n}}^{(\omega ; \beta ; \vec{a})}=\sum_{i=1}^{r} \omega n_{i} M_{\vec{n}-e_{i}}^{(\omega ; \beta+\omega \vec{a})}
$$

In particular, for $r=2$

$$
\Delta_{\omega} M_{n_{1}, n_{2}}^{\left(\omega ; ; ; a_{1}, a_{2}\right)}=\omega n_{1} M_{n_{1}-1, n_{2}}^{\left(\omega ; \beta+\omega ; a_{1}, a_{2}\right)}+\omega n_{2} M_{n_{1}, n_{2}-1}^{\left(\omega ; \beta+\infty ; a_{1}, a_{2}\right)} .
$$

Proof Changing $\beta$ for $\beta-\omega$ and applying the operator $\Delta_{\omega}$ to both sides of the generating function (7) for the case $r=2$ we obtain

$$
\begin{aligned}
\sum_{n_{1}=0}^{\infty} \sum_{n_{2}=0}^{\infty} \Delta_{\omega} M_{n_{1}, n_{2}}^{\left(\omega ; \beta ; a_{1}, a_{2}\right)} \frac{t_{1}^{n_{1}} t_{2}^{n_{2}}}{n_{1} ! n_{2} !}= & \left(\omega t_{1}+\omega t_{2}\right)\left(1-\frac{\omega t_{1}}{a_{1}^{\omega}-1}-\frac{\omega t_{2}}{a_{2}^{\omega}-1}\right)^{\frac{x}{\omega}} \\
& \times\left(1-\frac{\omega t_{1} a_{1}^{\omega}}{a_{1}^{\omega}-1}-\frac{\omega t_{2} a_{2}^{\omega}}{a_{2}^{\omega}-1}\right)^{\frac{-(\beta+x)}{\omega}} .
\end{aligned}
$$

By use of the generating function (7) the above equation gets the following form:

$$
\begin{aligned}
\sum_{n_{1}=0}^{\infty} \sum_{n_{2}=0}^{\infty} \Delta_{\omega} M_{n_{1}, n_{2}}^{\left(\omega ; \beta-\omega ; a_{1}, a_{2}\right)} \frac{t_{1}^{n_{1}} t_{2}^{n_{2}}}{n_{1} ! n_{2} !}= & \omega \sum_{n_{1}=0}^{\infty} \sum_{n_{2}=0}^{\infty} M_{n_{1}, n_{2}}^{\left(\beta, a, a_{2}\right)} \frac{t_{1}^{n_{1}+1} t_{2}^{n_{2}}}{n_{1} ! n_{2} !} \\
& +\omega \sum_{n_{1}=0}^{\infty} \sum_{n_{2}=0}^{\infty} M_{n_{1}, n_{2}}^{\left(\beta, a_{1}, a_{2}\right)} \frac{t_{1}^{n_{1}} t_{2}^{n_{2}+1}}{n_{1} ! n_{2} !}
\end{aligned}
$$

Changing $\beta$ for $\beta+\omega$ and then replacing $n_{1}$ with $n_{1}-1$ and $n_{2}$ with $n_{2}-1$ in the right side of equation we obtain

$$
\begin{aligned}
& \sum_{n_{1}=0}^{\infty} \sum_{n_{2}=0}^{\infty} \Delta_{\omega} M_{n_{1}, n_{2}}^{\left(\omega ; \beta ; a_{1}, a_{2}\right)} \frac{t_{1}^{n_{1}} t_{2}^{n_{2}}}{n_{1} ! n_{2} !} \\
& \quad=\sum_{n_{1}=0}^{\infty} \sum_{n_{2}=0}^{\infty}\left[\omega n_{1} M_{n_{1}-1, n_{2}}^{\left(\omega ; \beta+\omega ; a_{1}, a_{2}\right)}+\omega n_{2} M_{n_{1}, n_{2}-1}^{\left(\omega ; \beta+\omega ; a_{1}, a_{2}\right)}\right] \frac{t_{1}^{n_{1}} t_{2}^{n_{2}}}{n_{1} ! n_{2} !} .
\end{aligned}
$$

Finally, comparing the coefficients of $\frac{n_{1}^{n_{1}} t_{2}^{n_{2}}}{n_{1} ! n_{2} !}$, appearing on both sides of the above equation the desired result is obtained for the case $r=2$.

Remark 4.1 In the case $\omega=1$, the lowering operator for the $\omega$-multiple Meixner polynomials of the first kind coincide with the lowering operator for the multiple Meixner polynomials of the first kind which is given in [7]. For the proof of the $\omega$ type we consider a different approach, where the generating function plays an important role and the proof becomes simpler than the corresponding proof when $\omega=1$ [7, Theorem 2.4, p. 138]. 
Theorem 4.3 Let $\omega$ be a positive real number. The difference equation for the $\omega$-multiple Meixner polynomials $M_{\vec{n}}^{(\omega ; \beta ; \vec{a})}(x)_{|\vec{n}|=0}^{\infty}$ is given by

$$
\begin{aligned}
& L_{a_{1}}^{(\beta+2 \omega-r \omega)} L_{a_{2}}^{(\beta+3 \omega-r \omega)} \cdots L_{a_{r}}^{(\beta+\omega)} \Delta_{\omega} M_{\vec{n}}^{(\omega ; \beta ; \vec{a})}(x) \\
& \quad+\sum_{i=1}^{r} \omega n_{i} L_{a_{1}}^{(\beta+2 \omega-r \omega)} \cdots L_{a_{i-1}}^{(\beta+i \omega-r \omega)} L_{a_{i+1}}^{(\beta+(i+1) \omega-r \omega)} \ldots \\
& \quad \times L_{a_{r}}^{(\beta)} L_{a_{i}}^{(\beta+\omega)} M_{\vec{n}}^{(\omega ; \beta ; \vec{a})}(x)=0 .
\end{aligned}
$$

Proof Since $L_{a_{k}}^{(n)} L_{a_{m}}^{(n+\omega)}=L_{a_{m}}^{(n)} L_{a_{k}}^{(n+\omega)}$ for $n, a_{k}, a_{m} \in \mathbb{R}$, we obtain

$$
\begin{aligned}
L_{a_{1}}^{(\beta+2 \omega-r \omega)} L_{a_{2}}^{(\beta+3 \omega-r \omega)} \cdots L_{a_{r}}^{(\beta+\omega)}= & L_{a_{1}}^{(\beta+2 \omega-r \omega)} \cdots L_{a_{i-1}}^{(\beta+i \omega-r \omega)} \\
& \times L_{a_{i+1}}^{(\beta+(i+1) \omega-r \omega)} \cdots L_{a_{r}}^{(\beta)} L_{a_{i}}^{(\beta+\omega)},
\end{aligned}
$$

for $i=1, \ldots, r$.

Now applying $L_{a_{1}}^{(\beta+2 \omega-r \omega)} L_{a_{2}}^{(\beta+3 \omega-r \omega)} \cdots L_{a_{r}}^{(\beta+\omega)}$ to the lowering operator (11) and using the raising operator (10), we get the result.

Theorem 4.4 Let $\omega$ be a positive real number. The third order difference equation for the $\omega$-multiple Meixner polynomials $M_{\vec{n}}^{(\omega ; \beta ; \vec{a})}(x)_{n_{1}+n_{2}=0}^{\infty}$ of the first kind is given as

$$
\begin{aligned}
x( & x-\omega) \nabla_{\omega}^{2} \Delta_{\omega} y+x\left[\beta\left(a_{1}^{\omega}+a_{2}^{\omega}\right)+(x-\omega)\left(a_{1}^{\omega}+a_{2}^{\omega}-2\right)\right] \nabla_{\omega} \Delta_{\omega} y \\
& +\left[a_{1}^{\omega} \beta-x\left(1-a_{1}^{\omega}\right)\left(a_{2} \omega \beta-x \omega\left(1-a_{1}^{\omega}\right)\left(1-a_{2}^{\omega}\right)-a_{1}^{\omega} a_{2}^{\omega} \beta \omega\right] \Delta_{\omega} y\right. \\
& +\left[n_{1}\left(1-a_{1}^{\omega}\right)+n_{2}\left(1-a_{2}^{\omega}\right)\right] \omega x \nabla_{\omega} y \\
& +\omega(\beta-\omega)\left[\left[n_{1} a_{2}^{\omega}+n_{2} a_{1}^{\omega}-a_{1}^{\omega} a_{2}^{\omega}\left(n_{1}+n_{2}\right)\right]\right. \\
& \left.-\left(1-a_{1}^{\omega}\right)\left(1-a_{2}^{\omega}\right)\left(n_{1}+n_{2}\right) x\right] y=0 .
\end{aligned}
$$

Proof Considering the case for $r=2$ in Theorem 4.3, we have

$$
L_{a_{1}}^{(\beta)} L_{a_{2}}^{(\beta+\omega)} \Delta_{\omega} y+\omega n_{1} L_{a_{2}}^{(\beta)} y+\omega n_{2} L_{a_{1}}^{(\beta)} y=0,
$$

where $y=M_{n_{1}, n_{2}}^{\left(\omega ; \beta ; a_{1}, a_{2}\right)}(x)$, which gives the proof.

Corollary 4.5 The special cases of Theorem 4.1, Theorem 4.2 and Theorem 4.4, Theorem 4.4 when $\omega=1$ can easily be reduced to the results obtained in [7]. For instance, when $\omega=1$ in Theorem 4.1, we have the raising operator $L_{a_{i}}^{(\beta)}[\cdot]$ for any polynomial,

$$
L_{a_{i}}^{(\beta)}[y]=\frac{x}{1-a_{i}} \nabla y-\left[\frac{a_{i}(\beta-1)}{1-a_{i}}-x\right] y
$$

which is exactly the same formula as in [7, equation (2.4), p. 138]. 
When $\omega=1$ in Theorem 4.2 the lowering operator for multiple Meixner polynomials of the first kind has the following form:

$$
\Delta M_{\vec{n}}^{(\beta, \vec{a})}=\sum_{i=1}^{r} n_{i} M_{\vec{n}-e_{i}}^{(\beta+1, \vec{a})}
$$

which coincides with the formula in [7, equation (2.5), p. 138].

When $\omega=1$ in Theorem 4.4, the $(n+1)$ th order difference equation for multiple Meixner polynomials of the first kind becomes

$$
\begin{aligned}
& L_{a_{1}}^{(\beta+2-r} L_{a_{2}}^{(\beta+3-r)} \cdots L_{a_{r}}^{(\beta+1)} \Delta M_{\vec{n}}^{(\beta, \vec{a})}(x) \\
& \quad+\sum_{i=1}^{r} n_{i} L_{a_{1}}^{(\beta+2-r)} \cdots L_{a_{i-1}}^{(\beta+i-r)} L_{a_{i+1}}^{(\beta+(i+1)-r)} \cdots L_{a_{r}}^{(\beta)} L_{a_{i}}^{(\beta+1)} M_{\vec{n}}^{(\beta, \vec{a})}(x)=0,
\end{aligned}
$$

which reduces to the formula in [7, Theorem $2.5, p .139]$.

When $\omega=1$ in Theorem 4.4, the third order difference equation for multiple Meixner polynomials of the first kind is obtained as

$$
\begin{aligned}
& x(x-1) \nabla^{2} \Delta y+x\left[\beta\left(a_{1}+a_{2}\right)+(x-1)\left(a_{1}+a_{2}-2\right)\right] \nabla \Delta y \\
& \quad+\left[\left(a_{1} \beta-x\left(1-a_{1}\right)\right)\left(a_{2} \beta-x\left(1-a_{1}\right)\left(1-a_{2}\right)-a_{1} a_{2} \beta\right] \Delta y\right. \\
& \quad+\left[n_{1}\left(1-a_{1}\right)+n_{2}\left(1-a_{2}\right)\right] x \nabla y \\
& \quad+(\beta-1)\left[\left[n_{1} a_{2}+n_{2} a_{1}-a_{1} a_{2}\left(n_{1}+n_{2}\right)\right]-\left(1-a_{1}\right)\left(1-a_{2}\right)\left(n_{1}+n_{2}\right) x\right] y=0,
\end{aligned}
$$

which coincides with the formula in [7, Corollary 2.6, $p .139]$.

\section{1/2-Multiple Meixner polynomials of the first kind}

As we mentioned before for the case when $\omega=1, \omega$-multiple Meixner polynomials of the first kind reduce to the known multiple Meixner polynomials of the first kind. For the other values of $\omega$ we have new classes for multiple Meixner polynomials of the first kind where $\omega$ is positive real number. In this section we exhibit the case $\omega=1 / 2$ and state some relations for 1/2-multiple Meixner polynomials of the first kind such as weight functions, orthogonality conditions, the explicit form, the generating function and a third order difference equation.

1/2-Multiple Meixner polynomials of the first kind have the following weight functions:

$$
w_{i}^{(1 / 2 ; \beta)}(x)=\frac{\Gamma_{1 / 2}(\beta+x) a_{i}^{x}}{\Gamma_{1 / 2}(\beta) \Gamma_{1 / 2}(x+1 / 2)}=\frac{\Gamma(2(\beta+x)) a_{i}^{x}}{\Gamma(2 \beta) \Gamma(2(x+1 / 2))} \quad i=1,2, \ldots, r .
$$

By using these weight functions in (2), the orthogonality conditions for 1/2-multiple Meixner polynomials of the first kind can be written as

$$
\sum_{x=0}^{\infty} M_{\vec{n}}^{(1 / 2 ; \beta ; \vec{a})}\left(\frac{x}{2}\right)(-x)_{j} \frac{\Gamma(2 \beta+x) a_{i}^{x / 2}}{\Gamma(2 \beta) \Gamma(x+1)}=0, \quad j=0,1, \ldots, n_{i}-1
$$


The explicit form for 1/2-multiple Meixner polynomials can easily be obtained from (5) as follows:

$$
\begin{aligned}
M_{\vec{n}}^{(1 / 2 ; \beta ; \vec{a})}(x)= & \sum_{k_{1}=0}^{n_{1}} \cdots \sum_{k_{r}=0}^{n_{r}}\left(\begin{array}{l}
n_{1} \\
k_{1}
\end{array}\right) \cdots\left(\begin{array}{c}
n_{r} \\
k_{r}
\end{array}\right) \\
& \times \prod_{j=1}^{r}\left[\frac{\left(a_{j}^{1 / 2}\right)^{n_{j}-k_{j}}}{\left(2 a_{j}^{1 / 2}-2\right)^{n_{j}}}(-2 x)_{|\vec{k}|}(2 \beta+2 x)_{|\vec{n}|-|\vec{k}|}\right] .
\end{aligned}
$$

1/2-Multiple Meixner polynomials of the first kind have the following generating function:

$$
\begin{aligned}
\sum_{n_{1}=0}^{\infty} \cdots \sum_{n_{r}=0}^{\infty} M_{\vec{n}}^{(1 / 2 ; \beta ; \vec{a})}(x) \frac{t_{1}^{n_{1}} \cdots t_{r}^{n_{r}}}{n_{1} ! \cdots n_{r} !}= & \left(1-\frac{t_{1}}{2\left(a_{1}^{1 / 2}-1\right)}-\cdots-\frac{t_{r}}{2\left(a_{r}^{1 / 2}-1\right)}\right)^{2 x} \\
& \times\left(1-\frac{t_{1} a_{1}^{1 / 2}}{2\left(a_{1}^{1 / 2}-1\right)}-\cdots-\frac{t_{r} a_{r}^{1 / 2}}{2\left(a_{r}^{1 / 2}-1\right)}\right)^{-2(\beta+x)} .
\end{aligned}
$$

1/2-Multiple Meixner polynomials of the first kind satisfy the following third order difference equation:

$$
\begin{aligned}
x( & -1 / 2) \nabla_{1 / 2}^{2} \Delta_{1 / 2} y+x\left[\beta\left(a_{1}^{1 / 2}+a_{2}^{1 / 2}\right)+(x-1 / 2)\left(a_{1}^{1 / 2}+a_{2}^{1 / 2}-2\right)\right] \nabla_{1 / 2} \Delta_{1 / 2} y \\
& +\left[a_{1}^{1 / 2} \beta-x\left(1-a_{1}^{1 / 2}\right)\left(\left(a_{2} \beta\right) / 2-\left[x\left(1-a_{1}^{1 / 2}\right)\left(1-a_{2}^{1 / 2}\right)\right] / 2-\left[a_{1}^{1 / 2} a_{1}^{1 / 2} \beta\right] / 2\right] \Delta_{1 / 2} y\right. \\
& +\left[n_{1}\left(1-a_{1}^{1 / 2}\right)+n_{2}\left(1-a_{2}^{1 / 2}\right)\right] x / 2 \nabla_{1 / 2} y \\
& +(\beta-1 / 2) / 2\left[\left[n_{1} a_{2}^{1 / 2}+n_{2} a_{1}^{1 / 2}-a_{1}^{1 / 2} a_{2}^{1 / 2}\left(n_{1}+n_{2}\right)\right]\right. \\
& \left.-\left(1-a_{1}^{1 / 2}\right)\left(1-a_{2}^{1 / 2}\right)\left(n_{1}+n_{2}\right) x\right] y=0,
\end{aligned}
$$

where $\Delta_{1 / 2} f(x)=f(x+1 / 2)-f(x)$ and $\nabla_{1 / 2} f(x)=f(x)-f(x-1 / 2)$.

\section{Acknowledgements}

The second author of the manuscript, Illkay Elidemir would like to express her sincere gratitude to her supervisor Prof. Dr. Sonuç Zorlu Oğurlu for her guidance and continuous support. Elidemir is also grateful to Prof. Dr. Mehmet Ali Özarslan for his advice and support. Last, but not the least, we would also want to extend our appreciation to those who could not be mentioned here but played an important role for this work to be completed.

\section{Funding}

No funding.

\section{Availability of data and materials}

Data sharing not applicable to this article as no data sets were generated or analysed during the current study.

\section{Competing interests}

The authors declare that they have no competing interests.

Authors' contributions

The two authors contributed equally to the paper. All authors read and approved the final manuscript.

\section{Publisher's Note}

Springer Nature remains neutral with regard to jurisdictional claims in published maps and institutional affiliations.

Received: 29 December 2019 Accepted: 5 June 2020 Published online: 15 June 2020 
References

1. Aptekarev, A.I.: Multiple orthogonal polynomials. J. Comput. Appl. Math. 99, 423-447 (1998)

2. Arvesu, J., Coussement, J., Van Assche, W.: Some discrete multiple orthogonal polynomials. J. Comput. Appl. Math. 153, 19-45 (2003)

3. Chihara, T.S.: An Introduction to Orthogonal Polynomials. Mathematics and Its Applications, vol. 13. Gordon \& Breach, New York (1978)

4. Dayiragije, F.N., Van Assche, W.: Multiple Meixner polynomials and non-Hermitian oscillator Hamiltonians (2013). arXiv:1310.0982v2

5. Erdelyi, A.: Higher Transcendental Functions, Vol. I. McGraw-Hill, New York (1953)

6. Koekoek, R., Swarttouw, R.F.: The Askey-scheme of hypergeometric orthogonal polynomials and its q-analogue. Reports of the faculty of Technical Mathematics and Informatics No. 98-17, Delft (1998). arXiv:math/9602214v1

7. Lee, D.W.: Difference equations for discrete classical multiple orthogonal polynomials. J. Approx. Theory 150, 132-152 (2008)

8. Miki, H., Tsujimoto, S., Vinet, L., Zhedanov, A.: An algebraic model for the multiple Meixner polynomials of the first kind. J. Phys. A, Math. Theor. 45(32), 325205 (2012)

9. Mubeen, S., Rehman, A.: A note on k-gamma function and Pochhammer k-symbol. J. Inform. Math. Sci. 6, 93-107 (2014)

10. Nikiforov, A.F., Suslov, S.K., Uvarov, V.B.: Classical Orthogonal Polynomials of a Discrete Variable. Springer, Berlin (1991)

11. Nikishin, E.M., Sorokin, V.N.: Rational approximations and orthogonality. In: Translations of Mathematical Monographs, vol. 92. Am. Math. Soc., Providence (1991)

12. Van Assche, W.: Difference equations for multiple Charlier and Meixner polynomials. In: Elaydi, S., et al. (eds.) New Proress in Difference Equations, pp. 547-555. Taylor \& Francis, London (2003)

13. Van Assche, W., Coussement, E.: Some classical multiple orthogonal polynomials. J. Comput. Appl. Math. 127, 317-347 (2001)

\section{Submit your manuscript to a SpringerOpen ${ }^{\circ}$ journal and benefit from:}

- Convenient online submission

- Rigorous peer review

Open access: articles freely available online

- High visibility within the field

Retaining the copyright to your article

Submit your next manuscript at $\boldsymbol{\nabla}$ springeropen.com 